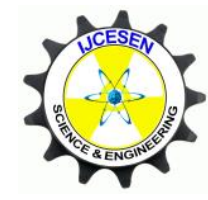

Copyright (C) IJCESEN
International Journal of Computational and

Experimental $\boldsymbol{S}$ cience and Engineering

(IJCESEN)

Vol. 4-No.1 (2018) pp. 16-23

http://dergipark.gov.tr/ijcesen

Research Article

\title{
Dynamic and quantitative Sensitivity analysis on the efficient development of tight oil
}

\author{
Jie Bai ${ }^{1 *}$, Huiqing Liu', Jing Wang1, ${ }^{1}$ Ling Xie ${ }^{2}$ and Xueping Li ${ }^{1}$ \\ ${ }^{1}$ Department of Petroleum Engineering, China University of Petroleum, Beijing, China \\ ${ }^{2}$ Optimization Petroleum Technologies, Inc \\ * Corresponding Author : jaybye@163.com \\ ORCID: 0000-0002-5722-8626
}

(First received 19 December 2017 and in final form 14 March 2018)

\section{Keywords}

tight oil

sensitivity analysis

threshold pressure gradient stress sensitivity

\begin{abstract}
The aim of this paper is to present dynamic and quantitative sensitivity analysis for the tight oil development with horizontal fracturing well. In order to better show the influence of different parameters on the production index in the development of tight oil, a set of correlations predict the oil production and effective swept scope are built correspondingly.

In this paper, 11 parameters are filtered after extensive investigation of typical characteristics of tight oil in China and America. The parameters include reservoir characteristics, threshold pressure gradient and stress sensitive effect, fracturing parameters etc. And then a group of orthogonal schemes were designed and simulated with the CMG IMEX Simulator. And then each five years' simulation results, including cumulative production, oil recovery and effective flow area are analyzed.

The analysis result shows that the initial reservoir pressure and well bottom hole pressure, which decides whether the energy is sufficient to drive oil flow, is the most significant factor to influence the tight oil cumulative production. Secondly, reservoir thickness and porosity influence whether the reservoir oil is rich. However, for the oil recovery, the contribution of fracturing is more essential, which directly determines whether it has industrial oil production. In addition, threshold pressure gradient and stress sensitivity, the distinct seepage characteristics between tight oil and traditional reservoirs, will further exacerbate the ultra-low permeability and seepage process. The most immediate effect of the non-Darcy rffect is that the swept scope range becomes smaller. In addition, a series mathematical model are also established for the development effect estimation through the use of multivariate regression, Through data validation, the quantitative model error ranges $5.45 \%$ from $9.29 \%$.
\end{abstract}

\section{Introduction}

With the stimulated fractured measure, tight formation develops different media, resulting in complex seepage[1,2,4,9,10,13]. Many factors affect oil production, and it's hard to predict and evaluate the tight oil production. In this paper, considering the characteristics of tight oil reservoirs (fracturing parameters[1,2,9,10,11,13], threshold pressure gradient $[3,12]$ and stress sensitive effect $[6,8,13]$,) a larger number of orthogonal experiment is carried on by simulating with CMG IMEX [5]. SPSS software [7] has also been used to study the sensitivity of well production and effective flow area of tight oil horizontal well. In addition, based on the sensitivity result, we established a set of reliable prediction models for oil production and effective flow area. These models are helpful for predicting the productivity of single well and the effective flow area, and then it can guide the optimization of horizontal well fracturing scheme and well pattern under different reservoir conditions.

\section{Parameter selection and research method}

Tight oil reservoir is difficult to develop due to the impact of many factors, including geological parameters, fracturing methods, non-darcy factors and well parameters. 


\subsection{Parameters selection (1) Reservoir characteristic}

The permeability of the tight oil reservoir is usually less than $0.10 \mathrm{mD}$ and the porosity is less than $10 \%$. The pore throat size reaches the micron and Nano levels. This basic permeability characteristic restricts the fluid flow of the reservoir. Despite the application of fracturing measures bring up effective industry production, the matrix permeability and porosity still occupy a large area in the fractured tight oil. $(\mathrm{Km}, \varphi)$

In addition, due to tectonic action, diagenesis, burial depth and other effects, reservoir pressure and reservoir effective thickness are also very imparity $(\mathrm{Pr}, \mathrm{H})$

For the different hydrocarbon source environment, the same as the tight reservoir, the fluid viscosity is also a key factor with big diversity.

\section{(2) Fracturing parameters}

The development of tight oil is mainly dependent on stimulated reservoir measure with large area, it supplies the effective technical support for tight reservoir. In specific, the length and fracturing space during the fracturing process have a very important effect on the tight oil production. Moreover, the choice of different proppant also resulted in a wide difference in fracture permeability. These fracturing parameters play an important role in the early high production of tight oil. (Kf, nf, Hf).

\section{(3) Non-Darcy factor}

Through the field practice and the laboratory pilot study' it is found that the fluid seepage in the tight oil does not conform to the regular Darcy seepage law. On the one hand, due to the tight structure of the reservoir and the development of different degrees of micro-cracks, there is a certain degree of stresssensitive effect - that is, with the increase in confining pressure, tight porosity and the permeability will decrease. Moreover, the different scale of the seepage media will occur in varying degrees of shrinkage deformation, including artificial fractures in the proppant compression will lead to fracture shrink or even closed. The stress sensitivity $[5,16]$ of the permeability is defined as follows

$$
k=k_{0} e^{-\alpha\left(p_{e}-p\right)}
$$

On the other hand, in the laboratory pilot study of the core of the displacement experiment, it is also found that the tight core displacement pressure and flow rate relation curve does not pass the origin (shown in Figure 1). Nevertheless, when the pressure difference is greater than a certain value, effective flow can be monitored. For this phenomenon, the concept of the threshold pressure gradient is proposed to characterize the non-Darcy characteristic of this low velocity seepage condition.

$$
v=\frac{k}{u}(\nabla p-G)
$$

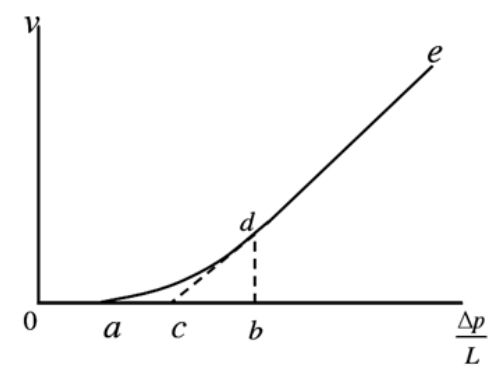

Figure 1. Tight core displacement pressure and liquid flow rate relation curve

\section{(4) Well parameters}

The production system of the well has a great influence on the development of the tight oil in the horizontal well. First, production pressure provides the most basic driving energy for the flow of oil. In particular, production pressure will further affect the stress-sensitive degree in tight reservoir. In this experiment, the horizontal well is designed to the constant pressure. Nevertheless, in the actual production there is still its production system control conditions. (BHP)

\subsection{Simulation model establishment}

It is very essential work to study the law of single well production and its influencing factors in tight oil reservoirs, which is also important for the overall deployment of tight oil development strategy formulation.

In this paper, the CMG IMEX simulator is used to complete the designed orthogonal scheme simulation and provide tight oil production simulation results

Numerical simulation model of single well in fine fracturing horizontal well. Horizontal section length $1300 \mathrm{~m}$. X, Y direction of the grid size were $20 \mathrm{~m}$, $20 \mathrm{~m}, \mathrm{Z}$ direction due to the consideration of different thickness of the reservoir, its size will be different.

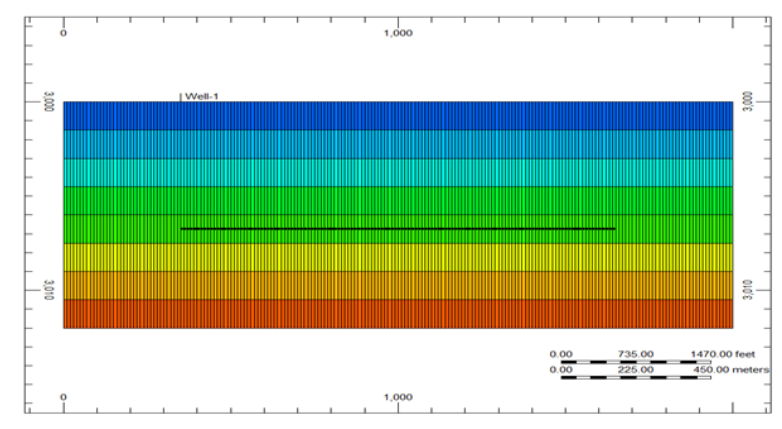

Figure 2. Basic tight oil model in CMG IMEX

\subsection{Orthogonal scheme design}


For the sensitive analysis of tight reservoirs, the conventional research involves the analysis of fracturing parameters. While the reservoir physical factors and non-Darcy characteristics are generally studied in terms of single reservoir characteristics. The reliability and applicability is poor, lacking of consideration of integrated parameters coupling influence.

Therefore, by researching the typical tight reservoirs in China and abroad including the characteristics parameters of the previous section, we filter the reasonable range of 11 factors in 5 level shown in the Table 1, the typical orthogonal experiment is designed and simulated by CMG IMEX. To each factor, the sensitivity is calculated by SPSS (variance analysis method) and the quantitative index is compared with each other. Furthermore, combined with the theory of seepage mechanics, the sensitivity result is analysed and explained.

Table 1. Orthogonal design of typical tight oil numerical model

\begin{tabular}{|c|c|c|c|c|c|}
\hline \multicolumn{7}{|c|}{ Formation property } \\
\hline Level & $\mathrm{Km}$ & $\Phi$ & $\mathrm{u}_{\mathrm{o}}$ & $\mathrm{Pr}$ & $\mathrm{H}$ \\
\hline$\%$ & $\mathrm{mD}$ & $/$ & $\mathrm{mPas}$ & $\mathrm{MPa}$ & $\mathrm{m}$ \\
\hline 50 & 0.02 & 0.05 & 7.5 & 20 & 8 \\
\hline 75 & 0.03 & 0.075 & 11.25 & 25 & 12 \\
\hline 100 & 0.04 & 0.1 & 15 & 30 & 16 \\
\hline 125 & 0.05 & 0.125 & 18.75 & 35 & 20 \\
\hline 150 & 0.06 & 0.15 & 22.5 & 40 & 24 \\
\hline
\end{tabular}

\begin{tabular}{|c|c|c|c|c|}
\hline \multicolumn{5}{|c|}{ NO-Darcy factors } \\
\hline Level & \multicolumn{2}{|c|}{ SP } & \multirow{2}{*}{\multicolumn{2}{|c|}{$\frac{\mathrm{G}}{\mathrm{Kpa} / \mathrm{m}}$}} \\
\hline$\%$ & \multicolumn{2}{|c|}{$\mathrm{MPa}^{-1}$} & & \\
\hline 50 & \multicolumn{2}{|c|}{0.5} & \multicolumn{2}{|c|}{300} \\
\hline 75 & \multicolumn{2}{|c|}{0.625} & \multicolumn{2}{|c|}{450} \\
\hline 100 & \multirow{2}{*}{\multicolumn{2}{|c|}{$\frac{0.75}{0.875}$}} & \multicolumn{2}{|c|}{600} \\
\hline 125 & & & \multicolumn{2}{|c|}{750} \\
\hline 150 & \multicolumn{2}{|c|}{1} & \multicolumn{2}{|c|}{900} \\
\hline \multicolumn{5}{|c|}{ Well and fracturing parameters } \\
\hline Level & BHP & $\mathrm{Nf}$ & $\mathrm{Kf}$ & $\mathrm{Hf}$ \\
\hline$\%$ & $\mathrm{MPa}$ & $\mathrm{n}$ & $\mathrm{D}$ & $\mathrm{m}$ \\
\hline 50 & 3 & 10 & 1 & 50 \\
\hline 75 & 4.5 & 15 & 1.5 & 75 \\
\hline 100 & 6 & 20 & 2 & 100 \\
\hline 125 & 7.5 & 25 & 2.5 & 125 \\
\hline 150 & 9 & 30 & 3 & 150 \\
\hline
\end{tabular}

\section{Simulation result analysis}

\subsection{Variance analysis principle and basic method}

The variance analysis [8] is mainly used to test the significance of the mean difference between two or more samples, thus determining whether the sample is taken from the population with the same mean. According to the variance analysis, combined with orthogonal test principle, sensitivity analysis can be carried out in SPSS software.

\subsection{Sensitivity analysis results}

Sensitivity analysis of different parental indexes was performed by using SPSS software by statistical analysis 11 factors and 5 level orthogonal test results. Different production indicators of the sensitivity of the parameters are neither same nor static. Therefore, we conducted a detailed comparison in each five years.

(1) Cumulative production

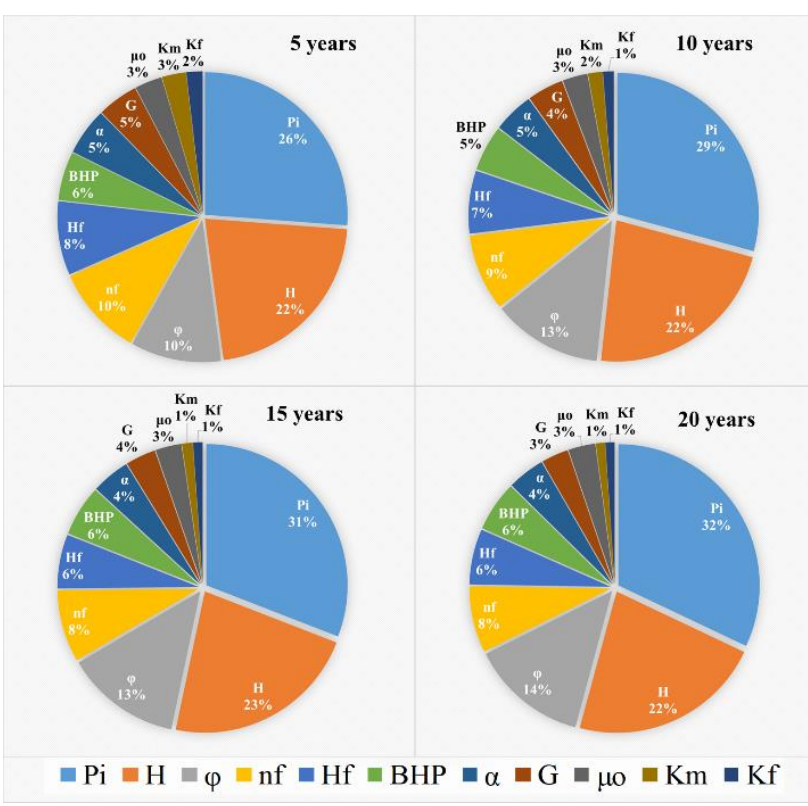

Figure 3. Contrast of cumulative production sensitive result in each five years

It can be seen that with time changing, the influence of each factor on cumulative production is not exactly same. The most important factors: $\operatorname{Pr}$ (nearly $30 \%$ ), H (nearly 13\%)and $\Phi$ (nearly 20\%). Pr (the original reservoir pressure) characterizes the reservoir energy adequacy. And $\mathrm{H}$ and $\Phi$ mainly reflect the oil richness in formation. Thus, it is found that adequate energy and material is the foundation for the sufficient production. Besides, the effect of threshold pressure gradient and stress sensitive effect is $10 \%$, which cannot be neglected either.

The influence of $\mathrm{H}$ (formation thickness) is not prominent to oil recovery, which is quite different with the cumulative production. At the same time, the fracturing parameters' ( $\mathrm{Hf}, \mathrm{nf}$ and $\mathrm{Kf}$ ) correlation with oil recovery is always greater than $20 \%$, especially in the early development or even up to highest $30 \%$. It reflects that the oil recovery is mainly related to the fracturing features, and the fractured zone is the dominate oil producing region, which directly affect the oil recovery.

However, with the lack of liquid supply in the fracturing area, the impact of fracturing parameters 
(2) Oil recovery

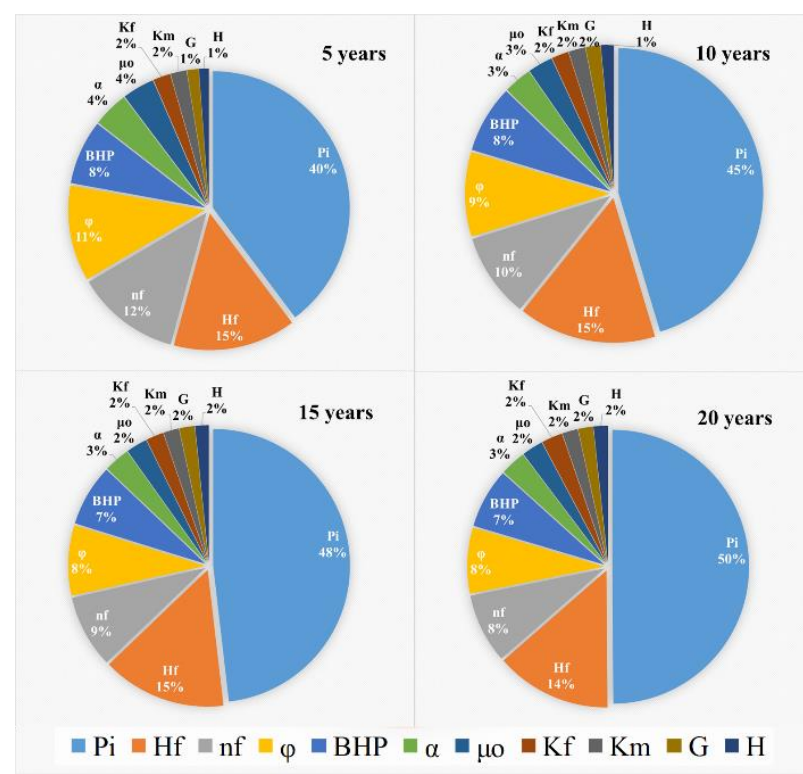

Figure 4. Contrast of oil recovery sensitive result in each five years

on the oil recovery has been reduced in the later stage of development. $\Phi$ accounts for about $10 \%$ proportion, and $\operatorname{Pr}$ (initial formation pressure) for about 5\%, reflecting negligible significance of oil abundance and formation energy for oil flow. And for the late stage, a reasonable change in oil supply zone occur from fracturing area to the matrix area. Under the influence of ultra-low permeability and threshold pressure gradient, the flow of oil in the matrix is demanding for the higher pressure gradient, that is why the effect of pressure becomes more pronounced during the later stage.

\section{(3) Effective flow area}
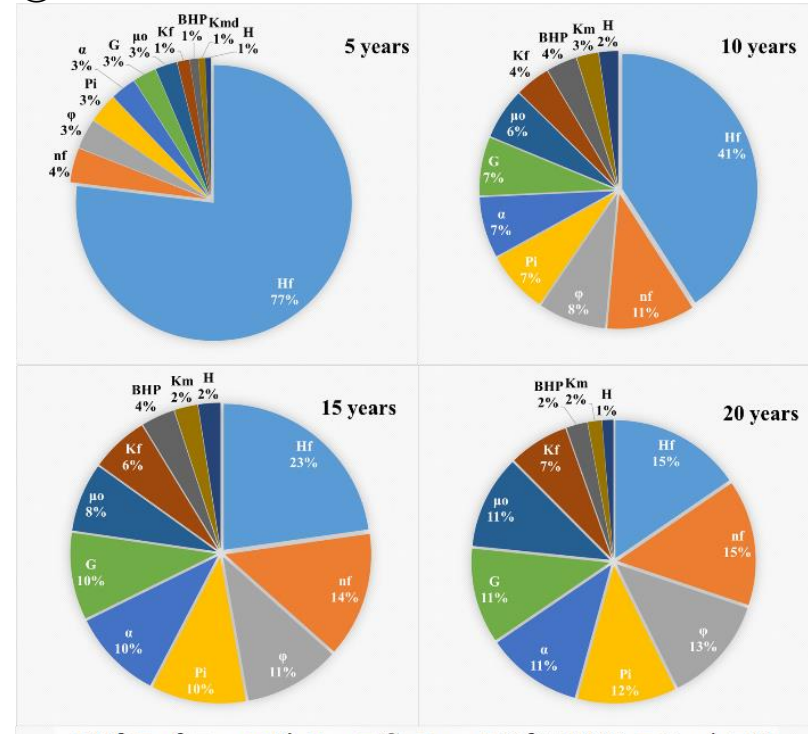

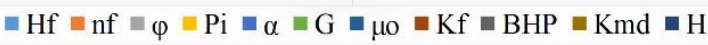

Figure 5. Contrast of Effective flow area sensitive result in each five years
The influence of each parameter on effective flow area changes significantly over time, especially the impact of fracture spacing and length ( $\mathrm{Hf}$ and $\mathrm{nf}$ ) from $80 \%$ gradually reduced to $32 \%$.

In the early stages of development, the flow occurred mainly in the fracturing area, which result in the excellent relevance with the effective flow area. But the effective flow area gradually expands to the close matrix between the artificial fracture, and then toward the outer matrix zone. To be specific When the flow in the close matrix between the artificial fracture dominates, the effect of the fracture spacing (equivalent to the fracture number) gradually increasing from $4 \%$ to $14 \%$. And in the same time, the correlation between flow area and the facture half length is bound to sharply reduce from $77 \%$ to $23 \%$. In the end, when the oil outer matrix zone flow dominates, the effect of the fracture half length decease further to $15 \%$, but the influence of fracture number changes a little. Besides, the impact of fracture permeability (Kf) is not significant still below $5 \%$.

The threshold pressure gradient (G)and stress sensitivity(SP) also showed a limited effect in the early stage, but a greater effect in the later stage. In the early stage, effective flow area is small, stresssensitive effects are limited in this area. In the same time, the pressure gradient is large enough, and it is easy to overcome the threshold pressure gradient. Their influence is not conspicuous with $6 \%$ proportion (shown in Fig.5).

But later, the expansion of the effective flow area will cause the pressure gradient to decrease, overcoming the threshold pressure gradient becomes more difficult. And the stress-sensitive's influence proportion reached $24 \%$, resulting in permeability deterioration in a wide area.

The effective flow distance along well direction has a great relationship with the length of the horizontal well. In the whole 20 years' production life, the influence of the parameters changed not much, among which stress sensitivity (SP), oil viscosity $(\mu \mathrm{o})$, matrix permeability (Kmd) and porosity $(\Phi)$ are the main factors. According to the pressure transmitting coefficient $(\eta)$ definition, the oil viscosity ( $\mu \mathrm{o})$, matrix permeability (Kmd) and porosity $(\Phi)$ is included in pressure transmitting coefficient $(\eta)$.The stress-sensitive effect on weakening permeability also directly affects the pressure transmitting coefficient $(\eta)$.

$$
\eta=\frac{k}{\phi \mu C_{t}}
$$


(4) Effective flow distance along well direction
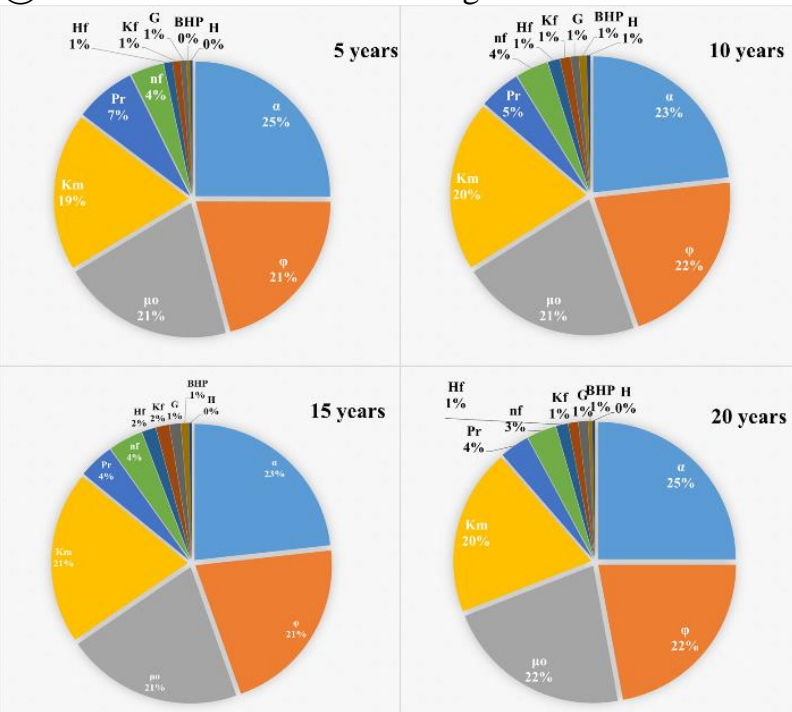

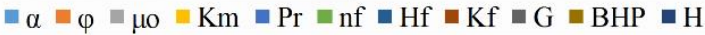

Figure 6. Contrast of effective flow distance along well direction sensitive result in each five years

(5) Effective flow distance along fracture direction

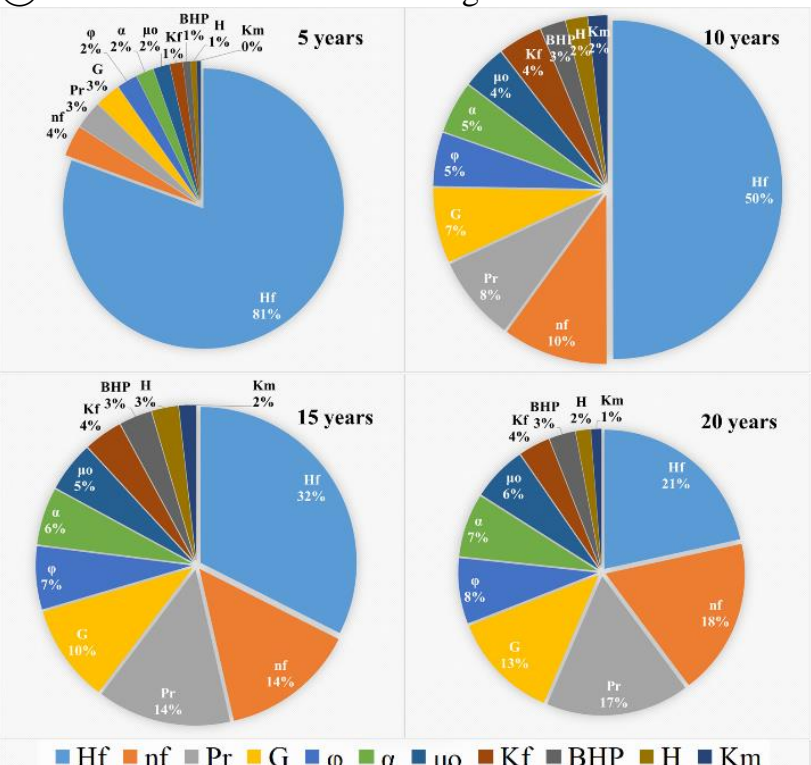

Figure 7. Contrast of effective flow distance along fracture direction sensitive result in each five years

Different from the flow along well direction, the fracture length plays the greatest role for the transmitting along well direction, seen from the Fig7. And its influence weight aggrandizes from the original $80 \%$ to $21 \%$. The fracture number proportion increases from $4 \%$ to $17 \%$, and the threshold pressure gradient part changed from $3 \%$ to $18 \%$. Stress-sensitive effects and initial pressure also increase to some extent.

\subsection{Sensitivity analysis of seepage mechanics theory}

In previous section, SPSS analysis software was used to evaluate the 11 factors' influence on the effective development in tight oil with horizontal fracturing well. According to the fracturing theory of fractured horizontal well rationality analysis. Due to the presence of multiple seepage zones in fractured reservoir, the impact of each factor is neither same nor static. In this section, the seepage process of different regions is analyzed by combining the results of sensitivity analysis.

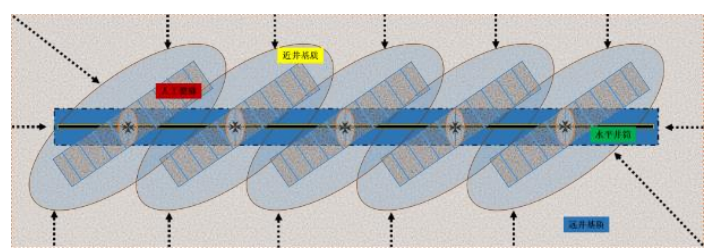

Figure 8. Fracturing horizontal well seepage process

a) Fracture - wellbore seepage stage

In the early stages, the oil in the high permeability induced fractures takes precedence to flow into the artificial main fracture, and then into the wellbore. At this stage, the effect of fracturing parameters is obvious. And the reservoir pressure is sufficient, short-term high production can be monitored.

b) Matrix - fracture seepage stage

Driven by sufficient pressure, the fluid in the matrix near the fracture and well begins to flow and Matrix between fracture contact with the fracture extensively, the oil of this zone is more likely to flow into the fracture, then through the fracture to the wellbore. Thus, at this stage, the fracture spacing has a greater effect on the effective flow area. And with the pressure transmission, the pressure gradient decreases in the whole reservoir, the influence of threshold pressure gradient and stress sensitive to the pressure transmission gradually emerging. Stress sensitivity decrease the effective permeability, and the threshold pressure gradient leads to difficulty of further pressure transmission. But the flow of fluid in the matrix region is still limited by the ultra-low permeability, and their contribution is not enough to maintain high and stable production.

c) Matrix - matrix seepage stage

In the later stage, the oil flows into the near-well area from the far-well area. The flow of the fluid is lowvelocity influenced by the poor permeability and non-Darcy effect (threshold pressure gradient and stress sensitivity). Specifically, the oil in the matrix area can effectively flow and generate pressure transmission, but their contribution to the production is still small. For the actual fracturing well in field, in the late matrix liquid supply stage, oil production can only be maintained at 3-4 $\mathrm{m}^{3} / \mathrm{D}$ or even lower. The effect of pressure-sensitive and threshold pressure gradients is still far greater on the low area than on production. 


\subsection{Establishment of multiple regression equation for horizontal fracturing well in tight oil reservoir}

In order to promote the practicability of the analysis results, the 20-year development effect indicators such as cumulative production, oil recovery and effective flow area etc. were selected to perform multivariate parameter regression ${ }^{[2,17,18]}$. Through mathematical modeling, a set of empirical formula is modeled to guide the evaluation of the development in tight oil with fracturing horizontal well.

Based on the sensitivity and positive and negative correlation of the 11 parameters screened in the Section 1, the basic exponential equation is used as the basic idea of regression.

The basic structure of the formula is shown in the Figure8. For the weight ratio of each factor, the positive and negative correlation is reflected by the exponential. Considering the theory of seepage mechanics, the relevant parameters are combined, such as the combination of permeability and viscosity ( $\lambda_{o}$-oil mobility $)$, and the formation pressure and bottom hole pressure combination ( $\Delta P$-drawdown pressure ).

$$
f(A, B, C \ldots)=\frac{\left(\frac{A}{B}\right)^{a} \times E^{e} \times(C-D)^{c}}{G^{g} \times F^{f}}
$$

Similarly, the production formula for different production times can be obtained as well.

The effective flow area is the most important parameter in the tight reservoir. Only by determining the effective area of the single horizontal well can the fracturing parameters be reasonably designed. In addition, the scheme of the well pattern also needs to be made on the basis of the effective flow range.

(1) Quantitative prediction model of effective flow area

$$
x=\frac{K_{m}{ }^{0.35} s p^{0.55} K_{\mathrm{f}}^{0.12} n_{\mathrm{f}}^{0.28} L_{\mathrm{f}}^{0.4} \Delta p^{0.4}}{\left(G^{0.42} \mu_{o}^{0.35} \phi^{0.33} h^{0.1}\right)}
$$

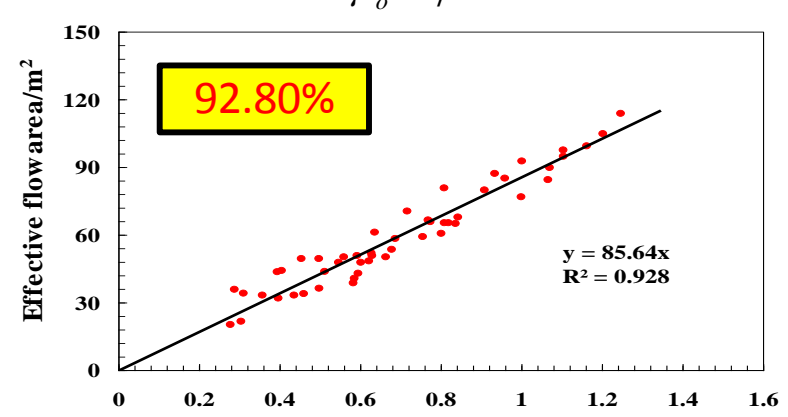

Figure 9. Effective flow area fitting curve

$$
A_{\mathrm{D}}=85.64\left(\frac{K_{m}}{\mu_{o}}\right)^{0.35} \frac{s p^{0.55}}{G^{0.42}} \frac{K_{\mathrm{f}}^{0.12} n_{\mathrm{f}}^{0.28} L_{\mathrm{f}}^{0.4}}{\phi^{0.33} h^{0.1}} \Delta p^{0.4}
$$

It can be seen that the main influencing factors are the mobility, reservoir pressure, fracture half-length and start pressure gradient.

(2) Quantitative prediction model of effective flow distance along well direction

$$
x=\frac{K_{m}^{0.5} s p^{0.9} n_{\mathrm{f}}^{0.07} \Delta p^{0.5}}{G^{0.05} \mu_{o}^{0.5} \phi^{0.48} h^{0.03}}
$$

In the scheme of the well pattern, it is necessary to carry out the design of the vector well pattern in consideration of the geological reality of the tight reservoir. In particular, the key parameters are the well spacing and row distance.

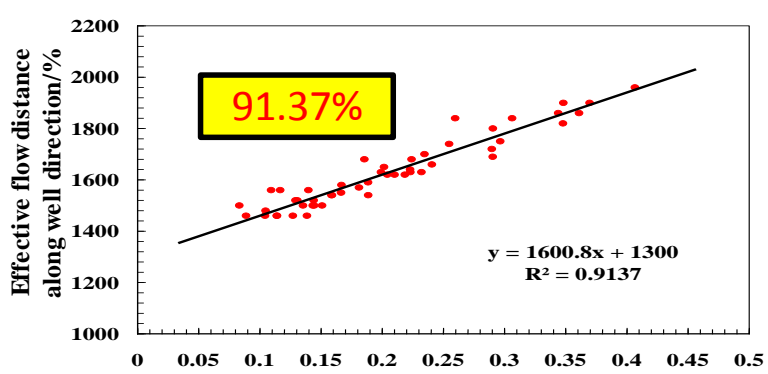

Figure 10. Effective flow distance along well direction fitting curve

$$
x=1600.8\left(\frac{K_{m}}{\mu_{o}}\right)^{0.5} \frac{s p^{0.9}}{G^{0.05}} \frac{n_{\mathrm{f}}^{0.07}}{\phi^{0.48} h^{0.03}} \Delta p^{0.15}+1300
$$

It can be seen that the main influencing factors in the direction of the shaft axis are mobility, porosity and formation pressure.

(3) Quantitative prediction model of effective flow distance along fracture direction $x=K_{m}{ }^{0.5} s p^{0.8} K_{\mathrm{f}}^{0.25} n_{\mathrm{f}}^{0.55} L_{\mathrm{f}}^{0.8} \Delta p^{0.75} /\left(\mu_{o}^{0.5} G^{0.88} \phi^{0.5} h^{0.25}\right)$

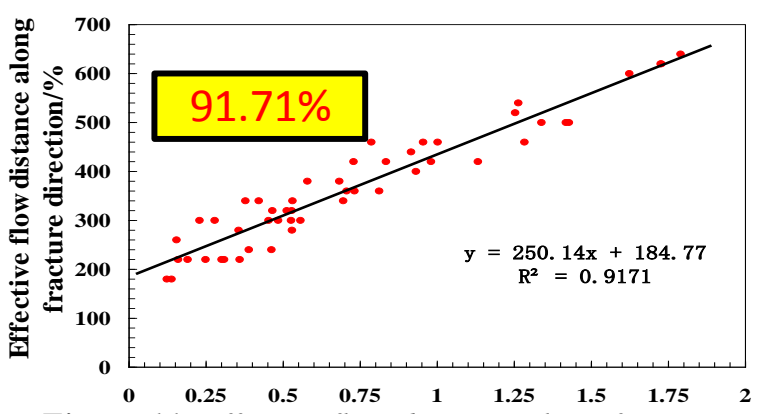

Figure 11. Effective flow distance along fracture direction fitting curve 


$$
Y=250.14\left(\frac{K_{m}}{\mu_{o}}\right)^{0.5} \frac{s p^{0.8}}{G^{0.88}} \frac{K_{\mathrm{f}}^{0.25} n_{\mathrm{f}}^{0.55} L_{\mathrm{f}}^{0.8}}{\phi^{0.5} h^{0.25}} \Delta p^{0.75}+184.77
$$

It can be seen that the main influencing factors in the direction of the fracture are the half-length of the fracture, the formation pressure, the threshold pressure gradient, the mobility and the porosity.

(4) Quantitative prediction model of cumulative oil production

From the previous part sensitivity analysis, it can be learned that the cumulative production and oil recovery have quite difference from the effective flow indicator. From the multiple regression, we also verify the rationality of the sensitivity difference based on the index of the parameters.

$$
x=\frac{K_{m}^{0.23} s p^{0.23} K_{\mathrm{f}}^{0.05} n_{\mathrm{f}}^{0.35} L_{\mathrm{f}}^{0.4} \Delta p^{1.35}}{G^{0.27} 0.152 \phi^{0.65} h^{0.9} \mu_{o}^{0.23}}
$$

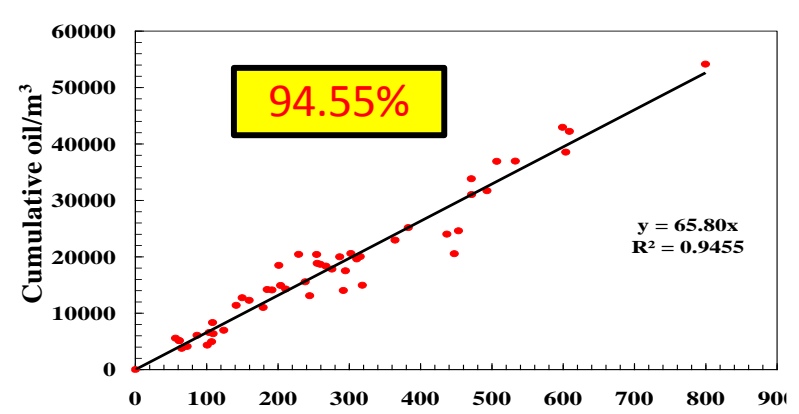

Figure 12. Cumulative oil production fitting curve

$$
\begin{array}{r}
V_{\mathrm{o}}=\left(\frac{K_{m}}{\mu_{o}}\right)^{0.23} \frac{s p^{0.23}}{G^{0.27}} \frac{K_{\mathrm{f}}^{0.05} n_{\mathrm{f}}^{0.35} L_{\mathrm{f}}^{0.4}}{0.152 \phi^{0.65} h^{0.9}} \Delta p^{1.35} \\
x=\frac{K_{m}^{0.14} K_{\mathrm{f}}^{0.06} n_{\mathrm{f}}^{0.32} L_{\mathrm{f}}^{0.55} s p^{0.4} \Delta p^{1.3}}{G^{0.25} \mu_{o}^{0.14} 120.5 \phi^{0.3} h^{0.17}}
\end{array}
$$

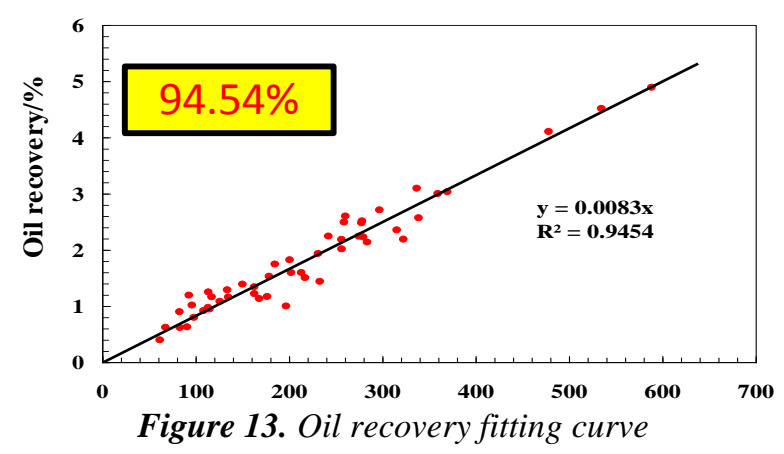

$$
E_{R}=\left(\frac{K_{m}}{\mu_{o}}\right)^{0.14} \frac{s p^{0.4}}{G^{0.25}} \frac{K_{\mathrm{f}}^{0.06} n_{\mathrm{f}}^{0.32} L_{\mathrm{f}}^{0.55}}{120.5 \phi^{0.3} h^{0.17}} \Delta p^{1.3}
$$

It can be seen that the main influencing factors of oil recovery are formation pressure, half-length of fracture, porosity and start pressure gradient.

Comparing the sensitivity ranking of each parameter in the previous section and the exponential index of the parameters in the regression model, better consistency can be observed.

\section{Conclusion}

In this paper, we analyzed the sensitivity of single well production in tight oil and stablished a set of reliable prediction models for oil production and effective flow area. The conclusions are as follows: 1. There are many influential factors in the development of tight reservoirs, which are mainly affected by the physical properties of tight reservoirs, fracturing parameters and non-Darcy factors. The relationship between the three is that the poor fluidity of the tight reservoir is the root cause of the fracturing, and the Darcy factor has a significant effect. on the flow after fracturing, and is reflected in the degree of development and the range 2. Due to the fracture and matrix reservoirs, there are different seepage stages with typical differences throughout the whole development period. In the early stage, the fracture are the most important factors, and in the later stages, the influence of the reservoir property and the non-Darcy factor becomes more significant.

3. For different development indicators (cumulative production, recovery, effective flow area), the weight of each factor will change. It is embodied that the most important factor for the effect of mulching is the reservoir physical parameters, which are mainly the formation pressure and viscosity, and the most important influence parameter is the fracturing parameters for the effective use range.

4. The impact of non-Darcy factor on the efficient development of tight oil from the early $20 \%$ to $35 \%$ of the late, which accounted for a large proportion of the early to start the main pressure gradient effect, and the corresponding impact of late pressure Further enhanced.

5. Based on the results of sensitivity analysis, a set of mathematic models for the prediction of accumulated oil, oil recovery and effective use of the range is established. The fitting degree of each model is more than $91 \%$, which is very important for the prediction of tight oil Production capacity and single well control area, and then developed for different reservoirs under the conditions of horizontal well fracturing program optimization and improvement, as well as the layout and optimization of horizontal well pattern has a guiding role. 


\section{Acknowledgement}

This paper presented in" ${ }^{\text {rd }}$ International Conference on Computational and Experimental Science and Engineering (ICCESEN-2017)"

And this study was supported by National Program on Key Basic Research Project (No. 2015CB250906) and Innovation Fund of CNPC (No. 2015D-5006-0204) in China.

\section{References}

[1]. Bai, B., Salehi, S., Nygaard, R., \& Aladasani, A. (2014, February 25). Optimizing the Development of Tight Oil Formations. Society of Petroleum Engineers.SPE-167742-MS. http://dx.doi.org/10.2118/167742-MS

[2]. Baig, M. T., Alnuaim, S., \& Rammay, M. H. (2015, June 4). Productivity Increase Estimation for Multi Stage Fracturing in Horizontal Wells for Tight Oil Reservoirs. Society of Petroleum Engineers. SPE178030-MS. http://dx.doi.org/10.2118/178030-MS

[3]. Baoquan, Z., Linsong, C., \& Fei, H. (2010, January 1). Experiment and Mechanism Analysis on Threshold Pressure Gradient with Different Fluids. Society of Petroleum Engineers. SPE-140678-MS. http://dx.doi.org/10.2118/140678-MS

[4]. Chen, H.-Y., \& Teufel, L. W. (2001, January 1). Understanding the Effects of Reservoir and Operating Parameters on Tight-Gas Production Decline. Society of Petroleum Engineers. SPE71066-MS. http://dx.doi.org/10.2118/71066-MS

[5]. CMG. IMEX User's Guide, Computer Modeling Group Ltd, 2012.

[6]. Cui, Q., \& Abass, H.H. (2016, May 5). Experimental Study of Permeability Decline in Tight Formations During Long-Term Depletion. Society of Petroleum Engineers.SPE-180257-MS. http://dx.doi.org/10.2118/180257-MS

[7]. Experimental design and Design-Expert, SPSS applications.

[8]. Lei, Q., Xiong, W., Yuang, J., Cui, Y., \& Wu, Y.-S. (2007, January 1). Analysis of Stress Sensitivity and Its Influence on Oil Production From Tight Reservoirs. Society of Petroleum Engineers. SPE111148-MS. http://dx.doi.org/10.2118/111148-MS

[9]. Li, X., Wei, H., Chen, B., Liu, X., Wang, W., \& Zhao, X. (2008, January 1). Multi-Stage Fracturing Stimulations Improve Well Performance in Tight Oil Reservoirs of the Chang qing Oilfield. International Petroleum Technology Conference. IPTC-12303MS. http://dx.doi.org/10.2523/IPTC-12303-MS

[10].Lin, M., Chen, S., Ding, W., Chen, Z. (John), \& Xu, J. (2015, May 1). Effect of Fracture Geometry on Well Production in Hydraulic-Fractured Tight Oil Reservoirs. Society of Petroleum Engineers. SPE167761-PA. http://dx.doi.org/10.2118/167761-PA

[11]. Roussel, N. P., \& Sharma, M. M. (2010, January 1). Optimizing Fracture Spacing and Sequencing in Horizontal Well Fracturing. Society of Petroleum Engineers.SPE-127986-MS. http://dx.doi.org/10.2118/127986-MS
[12]. Thomas, L. K., Katz, D. L., \& Tek, M. R. (1968, June 1). Threshold Pressure Phenomena in Porous Media. Society of Petroleum Engineers. SPE-1816-PA. http://dx.doi.org/10.2118/1816-PA

[13]. Wei, Y., et al., Determination of dynamic reserves of fractured-horizontal wells in tight oil reservoirs by multi-region material balance method. Petroleum Exploration \& Development, 2016. http://dx.doi.org/10.1016/S1876-3804(16)30057-X 